\title{
Of cuts and cracks: data analytics on constrained graphs for early prediction of failure in cementitious materials
}

\author{
Sanath Kahagalage ${ }^{1}$, Antoinette Tordesillas ${ }^{1,2, \star}$, Michał Nitka ${ }^{3}$, and Jacek Tejchman ${ }^{3}$ \\ ${ }^{1}$ School of Mathematics and Statistics, The University of Melbourne, Australia \\ ${ }^{2}$ School of Earth Sciences, The University of Melbourne, Australia \\ ${ }^{3}$ Faculty of Civil and Environmental Engineering, Gdańsk University of Technology, Poland
}

\begin{abstract}
Using data from discrete element simulations, we develop a data analytics approach using network flow theory to study force transmission and failure in a 'dog-bone' concrete specimen submitted to uniaxial tension. With this approach, we establish the extent to which the bottlenecks, i.e., a subset of contacts that impedes flow and are prone to becoming overloaded, can predict the location of the ultimate macro-crack. At the heart of this analysis is a capacity function that quantifies, in relative terms, the maximum force that can be transmitted through the different contacts or edges in the network. Here we set this function to be solely governed by the size of the contact area between the deformable spherical grains. During all the initial stages of the loading history, when no bonds are broken, we find the bottlenecks coincide consistently with, and therefore predict, the location of the crack that later forms in the failure regime after peak force. When bonds do start to break, they are spread throughout the specimen: in, near, and far from, the bottlenecks. In one stage leading up to peak force, bonds collectively break in the lower portion of the specimen, momentarily shifting the bottlenecks to this location. Just before and around peak force, however, the bottlenecks return to their original location and remain there until the macro-crack emerges right along the bottlenecks.
\end{abstract}

\section{Introduction}

There is no one-size-fits-all approach in data analytics. The best approach for analyzing data on current and historical behaviour of a system for the purposes of predicting future behaviour depends crucially on the problem context. When it comes to transmission and flow problems, techniques based on graphs or networks are arguably among the best in uncovering nonlinear and multiscale patterns from microstructural data (i.e., information on the individual units of the system), judging from their broad applications in transportation, telecommunication, supply chain and logistics, disease spread etc [1, 2]. Recently, network-based data analytics has also shown promise in studies of transmission patterns in granular media, but these were confined to unbonded grain assemblies [3-6].

The study reported here concerns the characterization and modelling of force transmission and failure propagation in cementitious granular materials (e.g., concrete, rock, bone, asphalt etc.) For a recent review of the stateof-the-art and challenges in the characterization and prediction of failure for this class of materials, see Lefort et al [7]. A promising new approach in damage mechanics, highlighted in [7], is the use of advanced statistical techniques to extract a length scale from spatial data on mesoscale damage events in acoustic emission experiments. They employed a spatial point pattern analysis that

\footnotetext{
^e-mail: atordesi@unimelb.edu.au
}

saw its first application to data sets for granular systems in [8]. Here, we similarly build upon a technique that has shown demonstrable promise in describing multiscale failure patterns from microstructural data in unbonded granular systems. Different from [7, 8], however, our aim here is to characterize and predict the ultimate macro-crack pattern. To achieve this, we go beyond descriptive analytics, since these use data mining to deliver insights into current and past behaviour only. Instead, we use network flow theory to uncover patterns from data that can help forecast in the pre-failure regime an aspect of a future event, viz., the location of the macro-crack.

In related past work, data from X-ray CT experiments and discrete element simulations, containing information at the level of individual grains [3-6], were analyzed using a network flow theory to identify bottlenecks and major flow pathways in force transmission for bonded and unbonded granular systems. This approach leads to an optimization problem in which the amount of flow through the network is maximised, considering all feasible paths from source to sink, for a given capacity distribution (i.e., the maximum flow units that can be transmitted across each edge of the network). The approach has shown promise in the prediction of: (a) the location and pattern of failure (localised versus diffused) [5, 6], and (b) force chains [6], without information on the contact forces that govern these mechanisms. Beyond prediction, these studies also delivered insights into the underlying structure and dynamics 


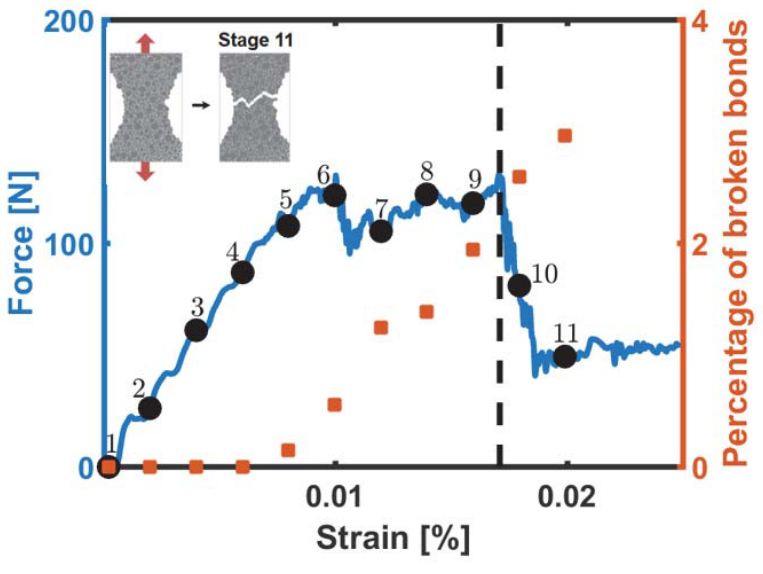

Figure 1. Data from DEM model. Strain evolution of the vertical tensile force in a 'dog-bone' concrete specimen submitted to uniaxial tension. We track behavior across 11 stages of loading: the strain state corresponding to the peak vertical force is shown by the vertical dashed line. A crack that splits the specimen into two pieces develops at stage 11. Right axis shows the evolution of damage as measured by the percentage of broken bonded contacts in the specimen (no damage occurs in stages 1-4).

of shear bands and force chains. In particular, findings in [5] for natural, synthetic and virtual granular assemblies demonstrated that flow bottlenecks emerge early in the loading history, progressively degrade, and persists in the location where the shear band eventually forms.

This study is part of a broader effort to investigate force transmission and failure in bonded granular media [9, 10]. In contrast to unbonded grains [5], force transmission pathways in brittle or quasi-brittle bonded granular materials remain essentially fixed prior to fracture. Accordingly, our aim is to determine the extent to which the distribution of bond strengths and contact topology per se govern the ultimate crack pattern. In particular, can the ultimate crack pattern be predicted even before any damage by bond breakage occurs? Because there is minimal loss, and ensuing frictional rearrangement, of contacts prior to peak stress in the sample, we neglect friction and any topological constraint that promotes frustration of rotations: the contact network sustains little "rewiring" and alteration of the available routes for transmission. Consequently, in this optimization problem, the flow through the network is regulated and maximised on highly constrained routes where the only change to the available pathways is through blockage of flow at edges where the corresponding bond breaks. Essentially no new routes form in the pre-failure regime where the prediction of failure matters.

\section{Data}

We discuss here the data set from a discrete element (DEM) model of an unconfined concrete specimen, described as a random heterogeneous one-phase material, subjected to quasi-static, uni-axial tension [9]. A linear normal contact model under compression was used. The contact tangential forces and normal forces satisfy

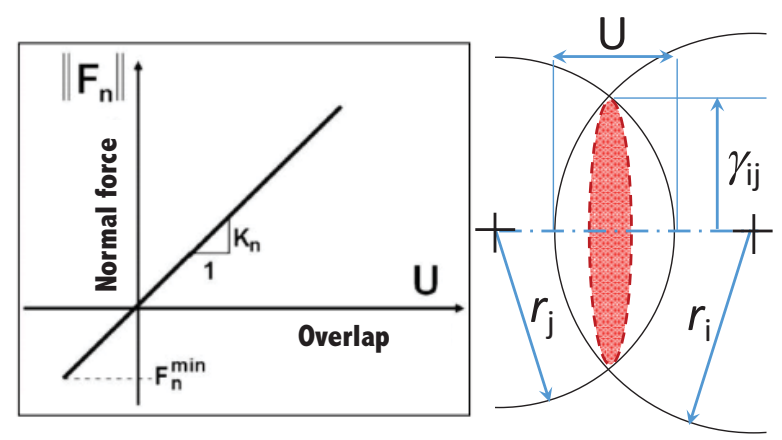

Figure 2. The normal tensile force depends on the overlap $U$; the bond is broken if this force reaches $F_{n}^{\min }$. The circular contact area (red) has radius $\gamma_{i j}$.

the cohesive-frictional Mohr-Coulomb equation using the inter-particle friction angle, permissible cohesive and tensile force. The bonded specimen has the dog bone shape with $0.15 \mathrm{~m}$ height, $0.10 \mathrm{~m}$ along the top and bottom, 0.06 $\mathrm{m}$ at mid-height and one layer of grains in depth. It contains 4942 grains with a wide distribution of radii: minimum is $2.7895 \times 10^{-4} \mathrm{~m}$, maximum is $5.3064 \times 10^{-3} \mathrm{~m}$.

The evolution with strain of the macroscopic force, along with the number of broken bonds, is shown in Figure 1. We analyse force transmission in 11 equilibrium stages: stages 1 to 9 are in the pre-peak force regime, while stages 10 to 11 are in the post-peak failure regime. The initial number of bonds is 12,350: bonds do not break until stage 5 from which the population of broken bonds steadily increases to around $2 \%$ just before peak force. The ultimate crack that forms in stage 11 span the 'neck' of the specimen (inset, Figure 1).

\section{Method}

We apply the method developed in $[3,5]$ to the data described in Section 2 as follows. For each equilibrium state, we formulate a network flow model of force transmission based on two input information: the contact network and the contact area over all the contacts. A network $G=\{V, E, C, s, t\}$ is constructed where: $G$ is a weighteddirected graph, $V$ is the set of intermediate vertices $i$ that represents the grains, $E$ is the set of edges $(i, j)$ that connect those vertices $i$ and $j$ in $V$ that correspond to grains in physical contact (bonded or unbonded frictional contact), $C=\left\{c_{(i, j)}, \quad(i, j) \in E\right\}$ is the set of scalar edge weights that represent the flow capacities (maximum units of flow that can be transmitted through the different edges in $E$ ), and $s$ and $t$ are two fictitious vertices that direct the flow from the source node $s$ to the sink node $t$.

Here the edge capacities $c_{(i, j)}$ represent the force bearing capacities of the corresponding contacts; hence $c(i, j)=c(j, i) \geq 0, \forall(i, j) \in E$. We choose the form of this capacity function to be in accordance with the DEM model from which the data, described in Section 2, was generated. As discussed in [9], and illustrated in Figure 2, the tensile force bearing capacity $\alpha_{i j}$ depends on the overlap $U=U_{i j}$ at the contact between the bonded spherical 
grains corresponding to nodes $i$ and $j$ :

$$
\alpha_{i j}= \begin{cases}k_{n} U>F_{n}^{\min } & \text { if the contact is bonded } \\ 0, & \text { if the contact is unbonded }\end{cases}
$$

where $k_{n}$ is the normal stiffness of the contact and $F_{n}^{\min }$ is the minimum tensile force as calibrated from uniaxial tension tests. The overlap is $U_{i j}=r_{i}+r_{j}-d_{i j}$, where $r_{i}$ and $r_{j}$ are the radii of the grains corresponding to the vertices $i$ and $j$, respectively, and $d_{i j}$ is the distance between the grains' centroids. Following eqn. 1, we propose an edge capacity $c(i, j)$ that depends solely on the size of the contact area, assumed here to be circular of radius $\gamma_{i j}$ :

$$
c_{i j}= \begin{cases}\pi \gamma_{i j}^{2}=\pi \times\left[r_{i}^{2}-\left(\frac{r_{i}-r_{j}+d_{i j}}{2}\right)^{2}\right] & \text { if the contact is bonded } \\ 0 . & \text { if the contact is unbonded }\end{cases}
$$

The larger the contact area, the stronger the bond, the higher the edge capacity.

Next we decide a direction of flow. Since the specimen is submitted to uniaxial tension in the vertical direction, we direct the flow from the top wall to the bottom wall, assigned as the source node $s$ and sink node $t$, respectively. Note that $G$ is symmetric, $c_{(i, j)}=c_{(j, i)}$; thus switching the source and sink assignments will not alter the flow. Following [5], we minimize boundary wall effects by connecting the top layer of grains (grains within two mean diameter away from the top wall) to $s$ while the bottom layer of grains (grains within two mean diameter above the bottom wall) to $t$. These artificial edges are assigned an infinite capacity so that only those contacts between constituent grains form candidates for bottlenecks.

To find the bottlenecks at each equilibrium state, we direct the maximum possible flow in $G$ from $s$ to $t$ by solving an optimisation problem known as the maximum flow-minimum cut [2]. For a given feasible flow $f$ of value $|f|$, the maximum flow problem is to find the maximum flow value $|f|_{\max }$ as follows. MAXIMIZE $|f|$ : $|f|=\sum_{i \in V} f(s, i)=\sum_{j \in V} f(j, t)$ subject to the following constraints:

1. Flow Capacity - For any edge $(i, j) \in E$, the flow cannot exceed its capacity: $f(i, j) \leq c(i, j)$.

2. Flow Conservation - For any vertex $i \in V$, the sum of the flows entering $i$ must be the same as the sum of the flows exiting $i: \sum_{i:(i, j) \in E} f(i, j)=$ $\sum_{j:(j, i) \in E} f(j, i)$.

The above is a dual of the minimum cut problem. Given a cut that partitions $G$ into two disjoint sets of vertices $S$ and $T$ with $s \in S, t \in T, S \cup T=V \cup\{s, t\}$, the minimum cut problem is to find that cut with the minimum capacity $c_{\text {min }}$ as follows. MINIMIZE $c: c(S, T)=\sum_{u \in S, v \in T} c(u, v)$. By the maximum flow-minimum cut theorem [2], the bottlenecks of force flow are the contacts corresponding to the edges in the minimum cut with capacity $|f|_{\max }=c_{\text {min }}$.

\section{Results}

There is no damage in the specimen across stages 1 to 4 , since bonds do not break until stage 5 (Figure 1). Dur- ing these initial stages of the loading when the specimen is damage-free (stages 1-4 of Figures 1 and 3), the bottlenecks coincide consistently with, and therefore predict, the location of the ultimate crack that forms in stage 11 after peak force (Figure 4). Damage is progressive from stage 5 onwards, as seen in the monotonic increase in the percentage of broken bonds (Figure 1). The initial sites of damage are spread throughout the specimen (stages 5 and 6 of Figure 3): here the bottlenecks remain in the same location as before, which is in the location of the crack that later forms in stage 11 (Figure 4). In the subsequent stages 7 to 8 , bonds on the lower part of the specimen collectively break, momentarily shifting the bottlenecks to this new location (stages 7 and 8 of Figures 3 and 4). Just before and around peak force, however, the bottlenecks return to their original location, reflecting the spread of damage there until the ultimate crack emerges right along the bottlenecks.

\section{Conclusions}

We developed a network flow approach for mining patterns from microstructural DEM data in a cementitious granular material, here a 'dog-bone' concrete specimen submitted to uniaxial tension. Force transmission bottlenecks, a subset of contacts that are prone to becoming overloaded beyond their capacity, can predict the location and pattern of the ultimate crack in the nascent stages of loading before damage initiates inside the specimen. The extent to which this data analytics technique can predict failure patterns in a range of multiphase concrete specimens and loading conditions (e.g., compression, 3-point bending tests $[9,10])$ will be reported in $[11]$.

\section{Acknowledgement}

This work was supported by the Australian Research Council (DP120104759), the US Army Research Office (W911NF-15-1-0527) and US Air Force Office of Scientific Research (FA2386-15-1-4059).

\section{References}

[1] R.K. Ahuja, T.L. Magnanti, J.B. Orlin, Network flows : theory, algorithms, and applications. (Englewood Cliffs, N.J. : Prentice Hall, c1993., 1993), ISBN 013617549X

[2] D.P. Bertsekas, Network optimization : continuous and discrete models., Optimization and neural computation series. 8 (Athena Scientific, 1998), ISBN 1886529-02-7

[3] A. Tordesillas, A. Cramer, D.M. Walker, Minimum cut and shear bands, in POWDERS AND GRAINS 2013: Proceedings of the 7th International Conference on Micromechanics of Granular Media (AIP Publishing, 2013), Vol. 1542, pp. 507-510

[4] Q. Lin, A. Tordesillas, Journal of Industrial and Management Optimization 10, 337 (2014)

[5] A. Tordesillas, S. Pucilowski, S. Tobin, M.R. Kuhn, E. Andò, G. Viggiani, A. Druckrey, K. Alshibli, Europhysics Letters 110, 1 (2015) 

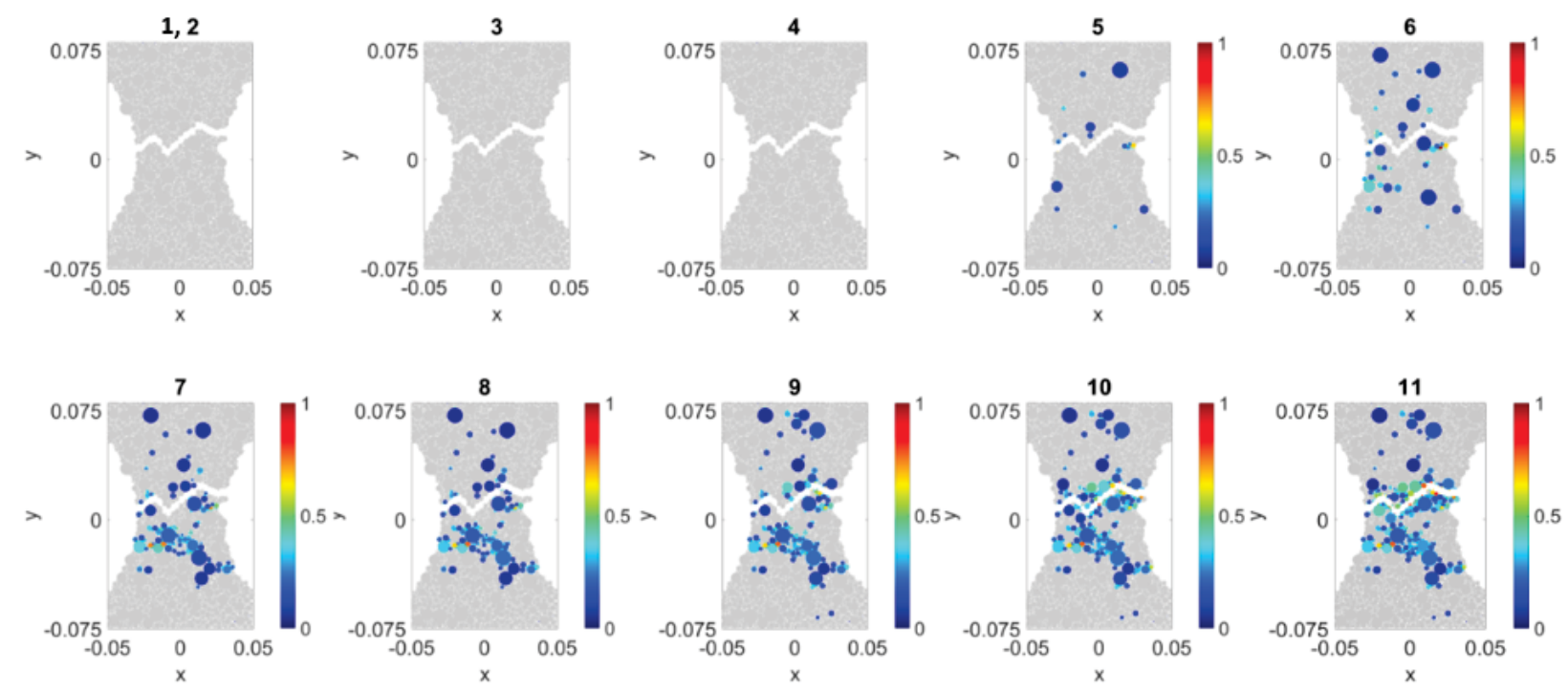

Figure 3. Spatial distribution of normalized damage sustained by individual grains, measured in terms of the percentage of number of broken bonded contacts relative to the initial number of bonded contacts (stages 1-4 are identical since no damage occurs across these stages). Solid gray grains are the grains with no broken bonds. The separation between the grains on either side of the crack that develops during failure (stage 11) is artificially increased to aid a visual comparison with the grains that sustained damage.
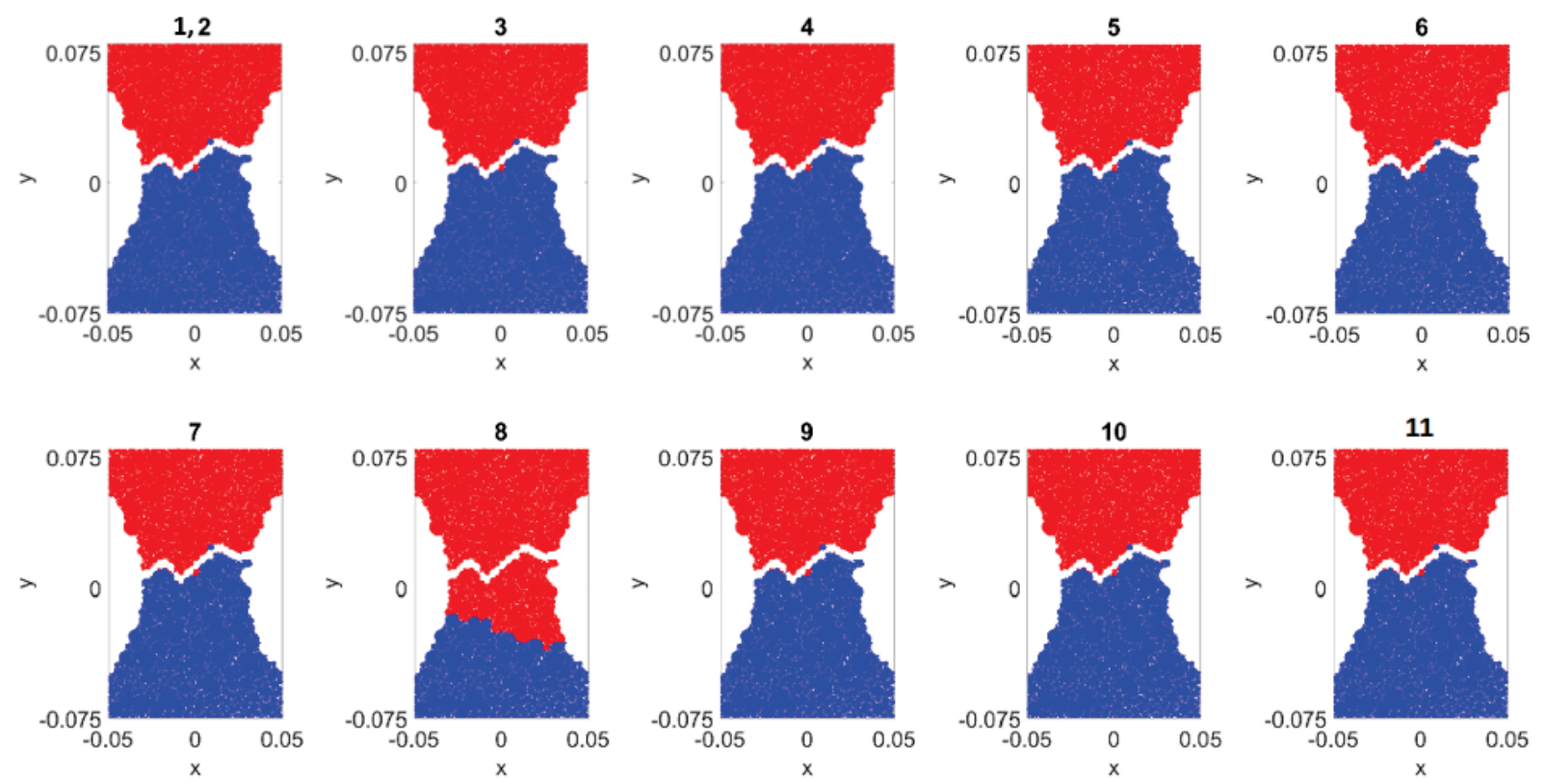

Figure 4. Spatial distribution of the predicted location of the crack, as identified from the bottlenecks in force transmission (red-blue interface). Grains on either side of the actual crack that later develops at stage 11 are artificially separated to aid visual comparison of the predicted crack location versus the actual crack location. Note the bottlenecks in stage 8 capture the spread of damage that momentarily dominates in the lower portion of the specimen just before peak force (recall stages 7-8 in Figure 1).

[6] A. Tordesillas, S.T. Tobin, M. Cil, K. Alshibli, R.P. Behringer, Physical Review E 91, 062204 (2015)

[7] V. Lefort, G. Pijaudier-Cabot, D. Grégoire, Engineering Fracture Mechanics 147, 449 (2015)

[8] A. Tordesillas, S. Pucilowski, L. Sibille, F. Nicot, F. Darve, Philosophical Magazine 92, 4547 (2012)
[9] M. Nitka, J. Tejchman, Granular Matter 17, 145 (2015)

[10] Ł. Skarżyński, M. Nitka, J. Tejchman, Engineering Fracture Mechanics 147, 13 (2015)

[11] A. Tordesillas, S. Kahagalage, M. Nitka, C. Ras, J. Tejchman (2017), in preparation 